\title{
Memory codes and negative transfer
}

\author{
EDWIN MARTIN \\ University of Michigan, Ann Arbor, Michigan 48.104
}

\begin{abstract}
A view of transfer phenomena in terms of composite memory codes, code independence, and modification of sampling probability over a stimulus-defined retrieval search space is discussed.
\end{abstract}

Suppose I once learned behavior B in situation A. Call that A-B learning. Suppose now for some reason I must learn new behavior $C$ in old situation $A$. Call that $A-C$ learning. The two learning tasks in sequence form the $\mathrm{A} \cdot \mathrm{B}, \mathrm{A}-\mathrm{C}$ transfer paradigm. Relative to an appropriate control paradigm, the A-B, A-C paradigm nearly always gives negative transfer. Suppose $B$ has always been your favorite theory about how a certain set of phenomena $A$ work. Suppose now it is obvious that theory B is wrong or woefully inadequate and that theory $\mathrm{C}$ is clearly better. You will have a much more difficult time changing over to theory $\mathrm{C}$ from theory $\mathrm{B}$ than a newcomer will have learning theory $\mathrm{C}$ without a past commitment to theory B. In short, there are certain forms of prior experience that render certain forms of current enlightenment very difficult. We call such prior-current relations negative transfer. Negative transfer is easily demonstrable in such diverse situations as problem solving, part-to-whole learning, clinical diagnosing, attitude formation, and perceptual identification of degraded or fleeting stimuli.

The problem is how to understand negative transfer; how to understand it, that is, in a general sense, not just as it occurs in rote paired-associate lists. How is it that old thoughts, attitudes, images, responses, whatever persist to the detriment of new learning? What happens to these old thoughts, attitudes, images, responses, whatever as new learning begins to succeed? A linear associationistic analysis is out of the question: An association is nothing more than a conditional probability, which makes it more a dependent variable than a theoretically useful construct. Moreover, no one yet has had a viable analytic idea about what either the argument $R$ or the conditionalizer $S$ should be in $P(R \mid S)$. And besides, in the one case where an associationistic theory came close to supporting a testable implication, it turned out to be dead wrong. I refer here to unlearning-interference theory and the easy counterdemonstration of stochastic independence of A-B and A-C associations (e.g., Martin, 1971). The notion of response suppression is equally untenable as a cornerstone for transfer theory: A response is suppressed if it is not emitted. The amount of suppression varies, of course, over certain experimental conditions, which is to say that performance in a transfer task or in a later memory test varies over certain experimental conditions. Knowing the regularities of such response likelihoods is not to know why they are like that, just as knowing how much and when the planet Venus dominates or is suppressed by the horizon is not to know celestial mechanics. What is required is a model, a model that entails structure and process. Such a model should organize the relevant phenomena in fewer statements than a complete listing of those phenomena.

Although degree of negative transfer is affected by many experimental variables, there are four particular transfer phenomena that seem fundamental for inducing a model of how transfer works:

(1) The intuitively reasonable idea that the nature of the response member of a stimulus-response pair is a determiner of which elements or components of the nominal stimulus become functional stimuli has been demonstrated experimentally (e.g., Merryman \& Merryman, 1971; Weaver, 1969). In other words, the nature of the memorial representation of a stimulus-response combination includes a representation of the response. The response code is not something at the end of a one-way chain; it is interactive with the stimulus code, a participating element of a combinatory code that represents the $A, B$ pair.

(2) If after A-B, A-C learning the $S$ is given a recognition test for $A \cdot B$, there is no acceptable evidence of retroactive interference due to interpolated A-C learning. This means that at some level of information representation in memory the $\mathbf{A}, \mathbf{B}$ combination is intact and undamaged. Delprato and Garskof (1968), Garsk of (1968), Goggin (1968, 1969), McGovern (1964), Postman (1965), and Sandak and Garskof (1967) have observed significant retroaction in matching tests, and Garskof and Bryan (1966) and Postman and Stark (1969) have observed nonsignificant retroaction in matching tests. These results, however, I consider unacceptable because of the problematic nature of such tests, involving as they do unassessed confusion and decision factors that need to be partialled out. My position on recognition tests for retroaction rests on an intuition that a properly analyzed yes-no recognition experiment would yield no evidence of retroaction, an intuition in accord with Bower and Bostrom's (1968) finding of neither proaction nor retroaction in short-term paired-associate recognition memory.

(3) If after A-B, A-C learning the $S$ is shown the $A$ stimulus and asked to recall the corresponding $B$ and $C$ responses as best he can. the $\mathrm{B}$ and $\mathrm{C}$ responses are 
recalled stochastically independently of each other (e.g.. Martin, 1971; Martin \& Greeno, 1972). The phenomenon of retroactive interference is that the unconditional probability of a B response is reduced. The phenomenon I refer to here is that the conditional probability of $B$ recall given $C$ recall is identical to the unconditional probability of $B$ recall. This means that there is a retrieval process of some sort that acts independently on intact $A, B$ and $A, C$ codes.

(4) We have recently demonstrated in our laboratory (Polzella \& Martin, in press) that if a learner changes his encoding of the nominal A stimulus in the second task of an $\mathbf{A}-\mathbf{B}, \mathrm{A}-\mathrm{C}$ paradigm, there is no negative transfer: performance is identical to that in an A-B, C.D control paradigm. To the extent that the $S$ does not change his stimulus code, he suffers negative transfer. In either case, however, we observe the independence between $A, B$ and $A, C$ codes I just mentioned as the third phenomenon of interest. What this means is that, if the $S$ incorporates into his code for the second-task A,C combination the same stimulus-code element he used in the first-task code for the A,B combination, he is in some sort of trouble. This trouble, however, does not entail damage to the first-task A,B code: Recognition is not impaired, as $I$ argued earlier, and independence between $A, B$ and $A, C$ still holds. The trouble that is negative transfer, then, must be in discriminating the $A, B$ and $A, C$ memory codes. It is reasonable that $A, B$ and $A, C$ codes should be more similar and, therefore, more resistant to discrimination when they contain a common component, as in the case where the $\mathrm{S}$ does not change the stimulus-code element when transferring to the second task. (For a fuller discussion of Points 1 and 4, see Martin, 1972.)

As you can see, I have mixed descriptions of empirical phenomena with my interpretation of them. I had no insidious intent. It is expositionally easier to couple theoretical assumptions with the phenomena they are based on.

The model for negative transfer I end up with goes like this: An A-B stimulus-response pair is represented in memory by a single integrative code. We can call this a Greeno code since it was Greeno (1970) who first injected some implicative power into this idea. The nature of such a composite code is interactively determined by its two components, which means that how the stimulus is coded can be affected by the response code. On encountering a second learning task, an A,C code is established. Establishing the A,C code involves forming a single representation in memory that combines $A$ and $C$ in some manner and then discriminating it from the $A, B$ code, a task that is difficult to the extent that the $A, B$ and $A, C$ codes are similar. Code similarity and the consequent amount of discrimination learning is what causes negative transfer.

So far, I have named a structure and a process. Respectively, they are what constitutes the learner's representation of his task, a composite code, and something called code discrimination. The nex specify what code discrimination is.

Specification of the nature of code discril relatively easy, for the simple reason alternative ideas are ruled out by two of phenomena: First, we know that establishing code does not obliterate the first code. This is subsequent recognition tests for A-B (Bower d 1968) and other demonstrations (e.g., Marti: 1971) that what was learned in the first tas means gone. Second, we know that in later tes $\mathrm{B}$ and $\mathrm{C}$ responses these responses ar stochastically independently of each othe 1971; Martin \& Greeno, 1972). It follows, the result of code discrimination in the second A-B, A-C paradigm is two noninteractive, ir composite codes, A,B and A,C, neither in structural impairment because of the existe other.

Now the facts that the A,B and A,C independent and that both can on occasion $b$ simultaneously to produce joint recall of suggests that stimulation by the nominal . initiates a retrieval search that must be : process. If the A,B code is selected, the respc the A,C code is selected, the response is $C$; included in the sample, we have joint recall c Accordingly, code discrimination must be a the distribution of sampling probability over similar codes, where by a similar-codes spac sampling domain in memory that encomp; composite codes that have components perce given nominal stimulus. The more similar is th to the $A, B$ code, that is, the closer togethe two codes in the sampling space, the more rad the sampling distribution be modified in support second-task success and, hence, negative is transfer. From a different persp sampling distribution over a domain of sin may be viewed as describing the level of $\mathrm{Hel}$ activity in the various regions of that do variance around the peak of that distributis viewed as the retrieval bandwidth, the criter: of acceptance of variations. Thus, after A-C the A-B, A.C transfer paradigm, the distribution will be peaked at the memory $]$ the A,C code. How sharply peaked the distrib be to insure response $C$ to stimulus $A$ depe similarity of the $A, B$ and $A, C$ codes, where $s$ : turn depends on B-vs-C response similarity ( 1972 , for further discussion) and on whether learner recoded the stimulus A (Polzella \& press).

According to this conversational and unpar model, retroactive interference occurs because of A-C learning the original A,B code has probability of being sampled, a probability th. exceed the sampling criterion. On the other 1 
are ways to alter the sampling distribution, one of which is to wait awhile and let it flatten out of its own accord, thus decreasing the likelihood difference between the $\mathrm{A}, \mathrm{B}$ and $\mathrm{A}, \mathrm{C}$ codes. The result in this case is spontaneous recovery. Also, according to this view, if after $A-B, A-C$ list learning the $S s$ were asked to free recall the $B$ and $C$ response members in the absence of the $A$ stimuli, one would expect $B$ and $C$ responses with common nominal stimuli to cluster together. This is because their composite codes would be more similar, that is, closer together in the sampling space, than would the composite codes for different pairs within the same list. This expectation has been approximated (Martin \& Mackay, 1970): Stimulus-defined response clustering is what occurs, not list-defined clustering.

The proposition that composite codes from the two tasks of a transfer paradigm are distinct and intact entities also jibes nicely with the now evident fact that retroactive interference is an item-specific effect (Birnbaum, 1972; Delprato, 1972; Merryman, 1971; Weaver, Duncan, \& Bird, 1972; Weaver, Rose, \& Campbell, 1971). In other words, neither associations nor responses are somehow set aside on a task membership basis; rather, the accessibility of the memorial representation of a given A-B contingency is altered only to the extent that a subsequent contingency must be learned that is specifically related to the original A. $B$ contingency.

Although I could continue to point out transfer phenomena that seem understandable from this single point of view, I will stop with the observation that, with ordinary experimental procedures, forward recall of a B response given its $\mathrm{A}$ stimulus is usually superior to backward recall of an A stimulus given its B response. The reason for this is that A-B learning entails code discrimination in a retrieval search space, or domain of memory, that is defined by the set of features that characterizes the initiating event, namely, the A stimulus. This discrimination process generally entails selectively partial utilization of the features that constitute a given stimulus. Consequently, when a memory search is suddenly initiated by the response member, the stimulus code element of the retrieved composite code is not sufficiently complete for the purpose of reconstructing the nominal stimulus.

As I pointed out earlier, Ss sometimes do and sometimes do not alter their encoding of the nominal stimulus in second-task A-C learning, and the evidence indicates a majority tendency toward not recoding the stimulus (Polzella \& Martin, in press). We do not know the conditions that determine the extent of the not-recoding strategy relative to the recoding strategy, but whatever those conditions might be, they should be of central importance because they decide whether the learner's subjective transfer paradigm is the A-B, A-C as intended by the $E$ or is the A-B.C.D as not intended by the E. But either way, the S's A.B and A.C codes are jointly intact and independent. even though differentially accessible. No associations are broken, and a given response is suppressed only in the sense that the form of probability density over a sampling space of $S-R$ combination codes has been altered by second-task learning.

According to the view I have been arguing for, the way in which various experimental variables affect the degree of negative transfer in the A-B, A-C paradigm boils down to this: The nature of the nominal stimulus and the nature of the responses determine how similar the corresponding $\mathrm{A}, \mathrm{B}$ and $\mathrm{A}, \mathrm{C}$ composite codes will be. We can add to this the interesting possibility that perhaps the combined features of the learning situation determine whether the learner will or will not change his functional encoding of the nominal stimulus, which in turn must further determine similarity between the A,B and $A, C$ codes. What then appears as negative transfer in the A-B, A-C paradigm relative to the A-B, C-D paradigm is the effect on second-task learning caused by the additional code discrimination peculiar to the A-B, A-C paradigm.

In summary, learning and transfer produce engrams. The question is: What are these engrams like and how does one select among them when the occasion arises? The basic facts of retrieval independence between A-B and $\mathrm{A}-\mathrm{C}$, of simultaneous permanence of both $\mathrm{A}-\mathrm{B}$ and $A \cdot C$, and yet of differential accessibility of responses B and $\mathrm{C}$ must be recognized together as a package. The corresponding notions of a composite code, of code independence, and of code discrimination through sampling distribution modification seem sensible as central elements in a relevant model. An approach that straightforwardly internalizes such simple dependent-variable-like concepts as $S-R$ association and response suppression, that is, an approach that mistakenly gives to these basically descriptive terms the status of axioms, must perforce be as complicated as the phenomena they are meant to explain.

\section{REFERENCES}

Birnbaum, I. M. General and specific components of retroactive inhibition in the A-B, A-C paradigm. Journal of Experimental Psychology, 1972, 93, 188-192.

Bower, G. H., \& Bostrom, A. Absence of within-list PI and RI in short-term recognition memory. Psychonomic Science. 1968. 10, 211-212.

Delprato, D. J. Pair-specific effects in retroactive inhibition. Journal of Verbal Learning \& Verbal Behavior, 1972. 11. 566-572.

Delprato, D. J., \& Garskof. B. E. Associative unlearning of A-B following $A-C$ or $A-B r$ interpolation. Journal of Experimental Psychology, 1968. 78.685-686.

Garskof, B. E. Unlearning as a function of degree of interpolated learning and method of testing in the A-B, A-C and A-B.C-D paradigms. Journal of Experimental Psychology, 1968. 76. 579.583.

Garskof. B. E.. \& Bryan. T. M. Unlearning as a function of degree of original learning and retention test. Psychonomic Science. $1966.6,391-392$.

Goggin. I. Retroative inhibition with different patterms of 
interpolated lists. Journal of Experimental Psychology, 1968, 76. 102-108.

Goggin, J. Retroactive interference with multiple interpolated lists. Journal of Experimental Psychology, 1969, 80, 483-488.

Greeno, J. G. How associations are memorized. In D. A. Norman (Ed.), Models of human memory. New York: Academic Press, 1970.

Martin, E. Verbal learning theory and independent retrieval phenomena. Psychological Review, 1971, 78, 314-332.

Martin, E. Stimulus encoding in learning and transfer. In A. W. Melton and E. Martin (Eds), Coding processes in human memory: Washington, D.C: Winston \& Sons, 1972.

Martin, E., \& Carey, S. T. Retroaction, recovery, and stimulus meaningfulness in the A-B. A-Br paradigm. American Journal of Psychology, 1971, 84, 123-133.

Martin, E., \& Greeno, J. G. Independence of associations tested: A reply to D. L. Hintzman. Psychological Review, 1972, 79, 265-267.

Martin. E., \& Mackay, S. A. A test of the list-differentiation hypothesis. American Journal of Psychology, 1970, 83, 311-321.

MoGovern. J. B. Extinction of associations in four transfer paradigms. Psychological Monographs, 1964, 78(16, Whole No. 593)

Merryman, C. T. Retroactive inhibition in the A-B, A-D paradigm as measured by a multiple-choice test. Journal of Experimental Psychology, 1971, 91, 212-214.
Merryman. C. T.. \& Merryman, S. S. Stimulus encoding in the $A-B^{\prime}, A X-B$ and the $A-B r^{\prime}, A X-B$ paradigms. Journal of Verbal Learning \& Verbal Behavior, 1971, 10.681-685.

Polzella, D. A.. \& Martin, E. Stimulus encoding in A-B. A-D transfer. American Journal of Psychology, in press.

Postman, L. Unlearning under conditions of successive interpolation. Journal of Experimental Psychology, 1965.70. 237-245.

Postman. L.. \& Stark, K. Role of response availability in transfer and interference. Journal of Experimental Psychology, 1969. 79. 168-177.

Sandak, J. M.. \& Garskof, B. E. Unlearning as a function of degree of interpolated learning in the A-B. A-C paradigm. Psychonomic Science, 1967. 7, 215-216.

Weaver, G. E. Stimulus encoding as a determinant of retroactive inhibition. Journal of Verbal Learning \& Verbal Behavior. $1969,8,807-814$

Weaver, G. E.. Duncan, E. M., \& Bird, C. P. Cue-specific retroactive inhibition. Journal of Verbal Learning \& Verbal Behavior. 1972, 11, 362-366.

Weaver, G. E., Rose, R. G., \& Campbell, N. R. Item-specific retroactive inhibition in mixed-list comparisons of the $A-B$, $A-C$ and $A-B, D-C$ paradigms. Journal of Verbal Learning \& Verbal Behavior, 1971, 10, 488-498

(Received for publication August 24, 1973.) 